\title{
The interface problem in interactive systems*
}

\author{
ABRAHAM WAKSMAN \\ Stanford Research Institute, Menlo Park, California 94025
}

\begin{abstract}
On-line computing implies a man-machine dialogue. It could be looked at as a communication channel with built-in constraints. We see a need to study in a systematic way how such constraints modify human behavior. Such studies will facilitate the needed specification for effective strategies in man-machine communication.
\end{abstract}

Human communication is characterized by interruptions, by a great many small errors, and by no apparent grammatical syntactic or semantic rules. Furthermore, in face-to-face communication, more than a single communication channel is involved.

In contrast, communication by means of computers is restricted to a keyboard or possibly to a graphical display of some sort. Computer communication language is formal in the sense that it is highly structured and allows no ambiguity in expressions. Furthermore, it is unforgiving in the sense that a deviation from the formal rules is not tolerated.

The quality of man-machine interaction is affected by even small changes, such as the speed at which the computer response is printed out. The difference in printout between 10 characters per second and 30 characters per second in its simplest case entails decisions such as: Should a problem solver request a latest problem status printout or should he defer such a printout and make do with what he thinks the status is?

There are few conclusive data on what other factors affect the quality of the interaction (for example, how intuition might mislead a designer of interaction systems) and how universal they are. It has been found that the quality of interaction does not seem to improve markedly when the ability for a user to interrupt is introduced. It was assumed that, for what is known as "scientific calculation," increasing the degree of freedom for a user to express intent might be a curse rather than a blessing. Also, for some well-defined clerical tasks, a well-structured and restricted formal interactive language was assumed to be desired.

Such assumptions have been proved wrong for a number of reasons, the main ones being that all systems tend to grow and that designers of languages and interface modes are not likely to foresee the ultimate use of their systems. A design approach currently being pursued holds great promise in alleviating some of the rigidity of present systems in tems of both expressing the user's intent and retrieving relevant material effectively.

This approach is becoming known as the "knowledge base systems approach." Such systems are characterized by a secondary data base that is separate from the main information store. It contains information about how

\footnotetext{
*This work was supported in part by the Office of $\mathrm{Naval}$
} Research under Contract N00014-71-0210. the data in the main store are intended to be used, the relationship between the user and the data, and the data sources. Within a limited context, such information represents a model of the user's universe of discourse. Such a model is useful in resolving semantic ambiguities and in making deductive inferences about the relevancy of sets of data for perusal and the type of response a particular user expects.

With all the great hope for such systems, most of them are at present experimental in nature and operate on tailored "toy" data bases. General principles or, for that matter, effective techniques for building such systems are only beginning to emerge.

The tendency in the research community in pursuing the development of knowledge-base systems is to fall back on what we know about human mental processes in information retrieval and problem solving-in particular, the notion of making associations between familiar items and the interpretation of meanings in terms of such associations.

Well-conceived psychological experiments might reveal more useful human processes that are applicable for incorporation into the internal computer model and that will contribute to increase the "power" of the man-machine interaction. Since a natural language discourse between man and machine is far in the future, it is reasonable to ask, for example, if a grammar based on the functional representation of elements is generally useful and practical to construct.

Can we construct formal languages based on how elements within the universe of discourse relate functionally to each other (i.e., more or less the way the human mind conceives their organization)? There is some evidence to the fact that such functional representation will appeal to a casual user since learning it will have a great intuitive appeal and will therefore require minimal effort.

Chapanis (1973) has studied some facets of the exchange of factual information when people solve a problem. He brings out a few unanswered questions that are relevant to our discussion: "How do communication patterns between man and machine vary with the person's ability?" "What sort of concessions will computers have to make for people at various levels of ability?" "How do communication patterns vary among different nationalities?" It has been accepted that communication patterns do differ markedly among 
different cultural groups. What sort of concessions will : computers have to make for such national and cultural groups?

Chapanis further observes that we really know very little about how actual meaning is conveyed in normal conversation. "At first glance natural interaction and communication between people convey the impression that they follow almost no grammatical, syntactical, or semantic rules, yet obviously there are rules, for meanings to get across and problems get solved very effectively at that." So the questions still stand, "How is meaning conveyed in natural conversation?" "What are the rules?" "How do we set about to study this problem and its implication to man-machine interaction?"

One possible avenue of attack is to consider experiments that will evaluate the amount of general knowledge used by people when answering simple questions. More specific evaluations could deal with the threshold or minimally related general knowledge needed in responding "intelligently" to simple questions. We further need to break down such knowledge into components, such as knowing why a given question was asked and knowing something about how the answer will be used.

The classical measure of goodness of a communication channel refers to the rate of information flow through the channel at the tolerable error rate. Such a measure considers only the fidelity of the transmission of strings of symbols through the channel. The meaning or the interpretation of the symbol strings is not considered part of the channel functions.

What should be a measure for a man-machine channel? How do we evaluate an information transfer rate through such a channel? Better yet, how do we set about optimizing such an information transfer?

In a man-machine communication, particularly for the casual user, it is very difficult to decide what constitutes a communication language barrier and what are the fundamental machine limitations-or even if such a separation is at all reasonable.

Let us consider an experiment consisting of two remotely located terminals essentially connected to each other through a computer program that ensures that communication between the two terminals follows the syntactic rules of some formal computer language.

Let us consider further that there are two humans, one at each terminal, who do not know each other. They are required to solve a problem that could be solved only by exchange of factual information through our "channel." This forces our two Ss to communicate via the terminals only and through the use of the formal computer language. We can monitor the length of time it takes to complete the task. We can monitor the number of exchanges during that period. We can monitor the strategies used by the two Ss to "size" each other, to understand the problem to be solved, and to familiarize themselves not only on how to use the formal communication language but also on how each is using the language. We can assume that after these initial stages, once the problem-solving session is in full swing, the communication channel is optimized. Such optimization is context dependent both on the problem at hand and the two problem solvers. Nevertheless, it represents a combination of the above-mentioned parameters that facilitates maximum information transfer.

The interface problem could then be defined as the problem of achieving a satisfactory level of communication between man and machine. For a steady, highly motivated user, such a problem is soon solved by virtue of his motivation and the fundamental system capabilities that he manages to summon.

A serious problem exists for the casual user. This is the person who has only an occasional need to interact with the system and for whom a complex interactive procedure is a serious deterent. Such a user finds it necessary to go through most of the initial familiarizing steps each time: the exploration of systems capabilities and the communication means of getting at them.

It seems that some implications as to process that a casual user is subjected to could be drawn from our two-terminal experiment. We can introduce a number of variations in the theme such as: $S A$ has the critical information for a problem solution needed by $S B$, but it is in a form that is not intelligible to $\mathrm{SA}$. He must operate under the instruction of S B in order to retrieve factual information needed by S B to solve a problem.

In summary, we feel that experiments as outlined, where the interplay between the "language" and the "systems capability" could be observed, will contribute toward optimizing man-machine communications by alluding to the degree required to each.

\section{REFERENCE}

Chapanis, A. The communication of factual information through various channels. Vol. 9. Information storage and retrieval. New York: Pergammon Press, 1973. Pp. 215-231. 